\title{
Pulling by Pushing, Slip with Infinite Friction, and Perfectly Rough Surfaces
}

\author{
Kevin M. Lynch \\ Matthew T. Mason \\ The Robotics Institute \\ and School of Computer Science \\ Carnegie Mellon University \\ Pittsburgh, PA 15213
}

\begin{abstract}
When one rigid object (the pusher) pushes another (the slider) across a horizontal support plane, Coulomb's law admits some surprising phenomena. First, it is possible to move the slider by moving the pusher away from the slider: pulling. Second, it is possible to obtain slip between the two objects even with an infinite coefficient of friction. Thus the common conception that infinite friction prevents slip is in error. This paper shows examples of the phenomena with both quasi-static and dynamic analysis. It also addresses implications for the concept of perfectly rough surfaces.
\end{abstract}

\section{Introduction}

Consider the problem of two rigid objects in frictional contact, supported by a horizontal planar surface, with gravity acting along the vertical. The motion of one object, the pusher, is given. The motion of the other object, the slider, is subject to Newton's laws. Frictional forces are governed by Coulomb's law. This paper constructs examples exhibiting two counterintuitive phenomena:

1. Pulling. The slider can maintain contact even when the pusher moves away from the slider.

2. Slipping with infinite friction. Even with an infinite coefficient of friction, slip can occur between the slider and pusher.

This second phenomenon has implications for the concept of perfectly rough contact. Usually a perfectly rough contact is defined as a contact that does not admit slip. Some treatments assume, erroneously, that this is equivalent to infinite friction. For example, Rutherford [11, page 41] states

"In the second case in which $\mu=\infty$ no sliding is possible and the surfaces are said to be perfectly rough."
Intuitively this seems correct, but we now see that the two concepts are not equivalent.

The paper begins with a review of the general method for solving frictional contact problems. Then we construct examples exhibiting the two phenomena. First we apply a quasi-static analysis, where forces of acceleration are assumed negligible, and then we follow with a full dynamic analysis. Finally we discuss alternative definitions of infinite friction and the relation to perfectly rough surfaces.

\section{Determining the motion of a pushed object}

The solution of frictional rigid-body contact problems presents some unusual difficulties. Under Coulomb's law, the force felt by a point contacting a surface must satisfy

$$
\left|f_{t}\right| \leq \mu f_{n}
$$

where $f_{t}$ is the tangential frictional component, $f_{n}$ is the normal component, and $\mu$ is the coefficient of friction. During sliding contact,

$$
\left|f_{t}\right|=\mu f_{n}
$$

and the frictional force is directed opposite the direction of sliding. Thus Coulomb's law does not directly specify contact forces. Rather, it imposes constraints that vary depending on the contact mode: whether the contact is being maintained, and whether the contact is sliding and in which direction. Frictional contact problems are solved by case analysis. For each contact mode, we determine whether forces and accelerations exist satisfying the simultaneous constraints of Coulomb's law, Newton's second law, and whatever kinematic constraints may be present. Each consistent set of forces and accelerations is deemed a solution to the problem. Problems with multiple solutions are ambiguous. Problems with no solutions are 


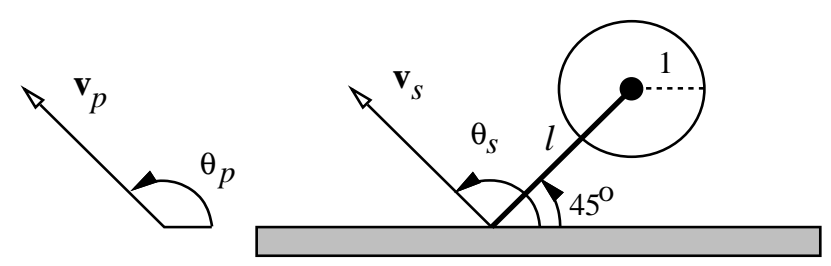

Figure 1: Example pushing problem.

inconsistent. More detailed descriptions are given by Lötstedt [8], Erdmann [5], Rajan et al. [10], Brost and Mason [3], Baraff [2], Dupont [4], and Wang et al. [12].

\section{Quasi-static pushing and pulling}

All of the examples in this paper are of the form illustrated in Figure 1. The pusher is a straight fence. The slider is a ring of radius 1 and uniform mass. The support force is distributed evenly about the ring, and the coefficient of support friction is uniform. Attached to the ring is a massless rod of length $l$ which does not contact the support surface. The slider is pushed at the free end of the rod. The rod makes an angle of 45 degrees with the fence. The coefficient of friction at the contact is $\mu$. The force $f$ applied by the pusher passes through the contact at an angle $\phi$. The pusher velocity is denoted $\mathbf{v}_{p}$, at an angle of $\theta_{p}$. The slider velocity at the rod endpoint is $\mathbf{v}_{s}$, at an angle of $\theta_{s}$. All angles are measured with respect to the fence.

We will say that a vector is directed into the slider if it has a nonnegative component along the outward-pointing normal of the fence, that is, if the angle is in the closed interval $\left[0^{\circ}, 180^{\circ}\right]$. Otherwise we will say that it is directed away from the slider. Force vectors are drawn with filled arrowheads and velocity and acceleration vectors are drawn with open arrowheads.

Velocities will sometimes be represented as velocity centers. The velocity center is the point in the plane about which the motion is a pure rotation. Similarly, initial accelerations will sometimes be represented as acceleration centers.

Now suppose that the slider motion is slow enough that inertial forces are negligible compared with contact forces. Then a solution obtains whenever the support frictional forces balance the pusher contact force. Consider Figure 2, for example. For the velocity center shown, the frictional support forces acting on the slider are precisely balanced by the pusher force $\mathbf{f}$ passing through the contact. For a pusher velocity $\mathbf{v}_{p}$ equal to the solution slider velocity $\mathbf{v}_{s}$, and a large enough coefficient of friction $\mu$, we have a solution. There is nothing unusual about this solution. Since the pusher velocity $\mathbf{v}_{p}$ is directed into the slider, we have pushing, not pulling.

But consider the example of Figure 3, where the applied

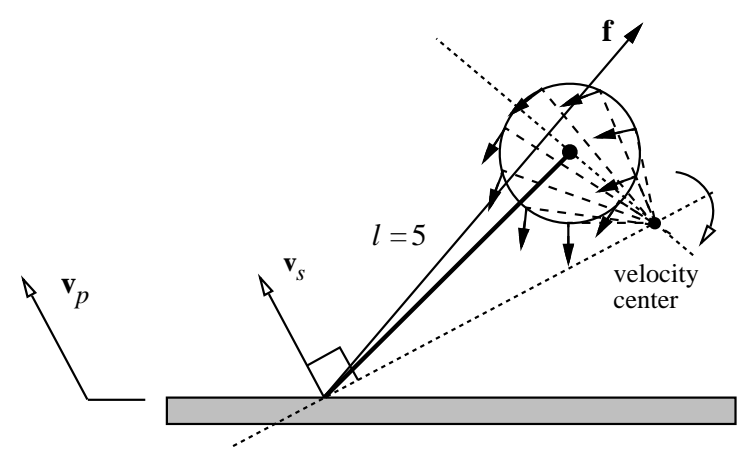

Figure 2: Quasi-static pushing.

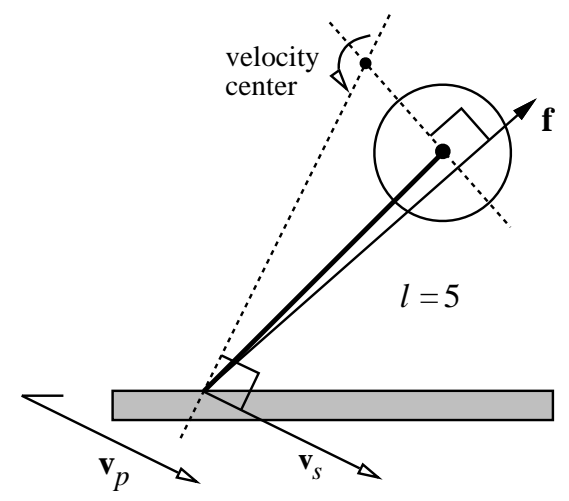

Figure 3: Quasi-static pulling.

force passes closely on the other side of the ring center. Here a solution is obtained for $\mathbf{v}_{p}$ equal to $\mathbf{v}_{s}$ but directed away from the slider: pulling. Note that a second solution is that the slider simply breaks contact and does not move. This problem is ambiguous, as are all of the pulling examples that we will construct.

To show that the solutions shown in Figures 2 and 3 really are solutions, we integrate the support frictional forces around the ring and show that the total frictional force the slider applies to the support equals $f$. We use two coordinate systems (Figure 4), one system $x-y$ aligned with the pusher, and a primed system $x^{\prime}-y^{\prime}$ aligned with the applied force but centered on the slider.

With some modifications, Goyal [6] gives the following expressions for frictional force and torque applied by the ring to the support surface in the primed system:

$$
\begin{aligned}
& f_{x^{\prime}}=0 \\
& f_{y^{\prime}}=\operatorname{sgn}(v) \frac{W}{2 \pi} \int_{0}^{2 \pi} \frac{1+\frac{\omega}{v} \cos \theta}{\sqrt{1+\left(\frac{\omega}{v}\right)^{2}+2 \frac{\omega}{v} \cos \theta}} d \theta \\
& \tau_{0^{\prime}}=\operatorname{sgn}(v) \frac{W}{2 \pi} \int_{0}^{2 \pi} \frac{\frac{\omega}{v}+\cos \theta}{\sqrt{1+\left(\frac{\omega}{v}\right)^{2}+2 \frac{\omega}{v} \cos \theta}} d \theta
\end{aligned}
$$




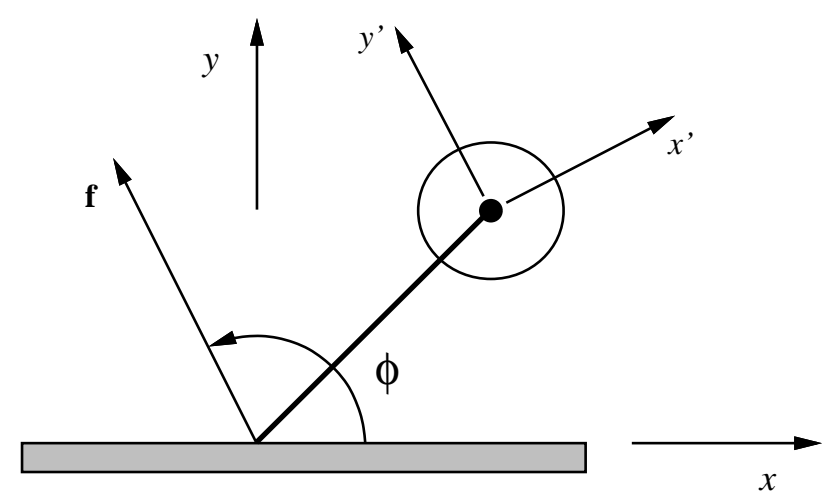

Figure 4: Coordinate conventions.

where $W$ is the slider weight multiplied by the support friction coefficient, $\omega$ is the slider angular velocity, and $v$ is the velocity of the slider center along the $y^{\prime}$ axis. (The velocity of the slider center along the $x^{\prime}$ axis is zero. The velocity center will fall a directed distance $-v / \omega$ along the $x^{\prime}$ axis.) The integrals are elliptic integrals which could be reduced to normal form, as in [6].

The frictional force and torque measured in the unprimed system is:

$$
\begin{aligned}
f_{x} & =f_{y^{\prime}} \cos \phi \\
f_{y} & =f_{y^{\prime}} \sin \phi \\
\tau_{0} & =\tau_{0^{\prime}}+l f_{y^{\prime}} \sin \left(\phi-45^{\circ}\right)
\end{aligned}
$$

For quasi-static balance the torque about the contact point must be zero:

$$
\tau_{0}=0
$$

Equation 7 implicitly defines a velocity center as a function of the force angle $\phi$. In principle, we could solve equation 7 for $\omega / v$ as a function of $\phi$. The quantity $-v / \omega$ gives the velocity center as a directed distance along the $x^{\prime}$-axis and the sign of $-\tau_{0}$, gives the rotation sense.

In practice, the relation of the pusher force $\mathbf{f}$ to the slider motion can be obtained numerically. We can also employ the limit surface of Goyal et al. [7] to represent this relation. The limit surface is the locus of $\left(f_{x}, f_{y}, \tau\right)$ arising from support friction during all possible slider motions. It is a closed, convex, two-dimensional surface enclosing the origin. Goyal showed that if a slider motion $\left(v_{x}, v_{y}, \omega\right)$ causes the slider to apply a force $\left(f_{x}, f_{y}, \tau\right)$ to the support, then $\left(v_{x}, v_{y}, \omega\right)$ is parallel to the outward-pointing normal of the limit surface at $\left(f_{x}, f_{y}, \tau\right)$.

For the present case, the limit surface can be reduced to a curve in the plane. The limit surface is constructed with forces measured in the $x-y$ coordinate system and torques measured about the pushing contact. But we are interested only in those motions producing no torque about this point.

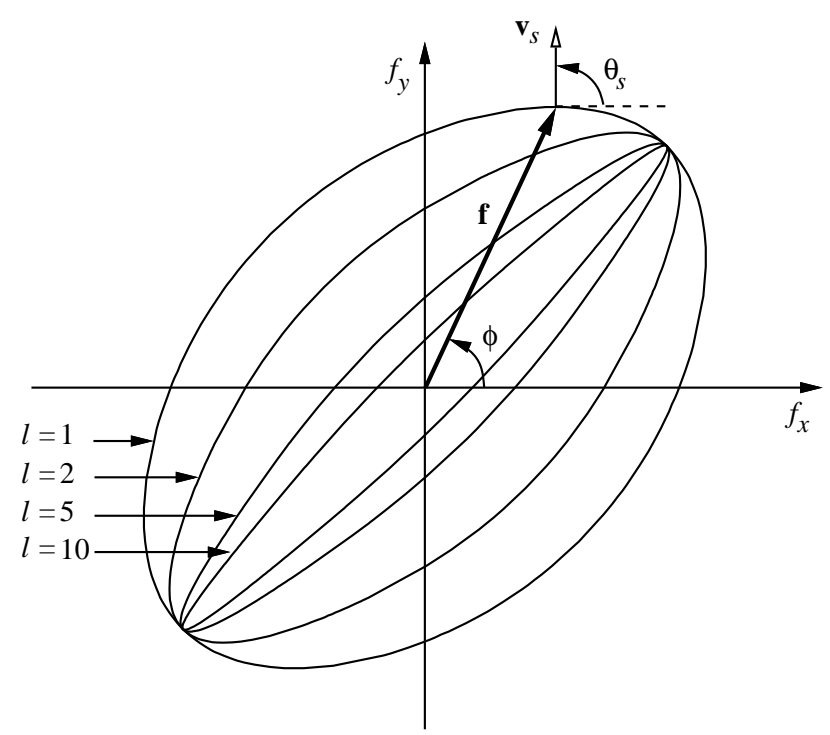

Figure 5: Limit curves for different rod lengths.

Therefore, we intersect the limit surface with the plane $\tau_{0}=0$ to obtain a limit curve representing the locus of frictional forces corresponding to possible solutions of the slider motion. Now choose $f$ to be one such force on the limit curve, and let $\mathbf{v}_{s}$ be the corresponding velocity of the slider contact point. Then $\mathbf{v}_{s}$ is normal to the limit curve at f.

Figure 5 shows limit curves for several different values of $l$. (The limit curves may appear to be elliptical, but they are not.) As $l$ approaches zero, the limit curve approaches a circle. As $l$ approaches infinity, the limit curve approaches a line segment aligned with the rod. The force $f$ and the velocity $\mathbf{v}_{s}$ are parallel only at local extrema of the limit curve. The force which the support friction can resist is maximized at $\phi$ equal to 45 degrees and 225 degrees, which correspond to pure lengthwise translations of the slider, and minimized at $\phi$ equal to 135 degrees and 315 degrees. At these four force angles, $\theta_{s}$ is equal to $\phi$.

\subsection{Friction cones and velocity cones}

Coulomb's law implies a simple constraint on the total force acting at a point contact: the force must act on a line through the contact and inside a friction cone defined by the directed lines which make an angle of $\tan ^{-1} \mu$ with the contact normal. Similarly, we can define the velocity cone [9] to be a constraint on the possible velocity directions of the slider contact point. Each force inside the contact friction cone is mapped by the limit curve to a corresponding contact point velocity angle $\theta_{s}$. These velocity vector directions sweep 

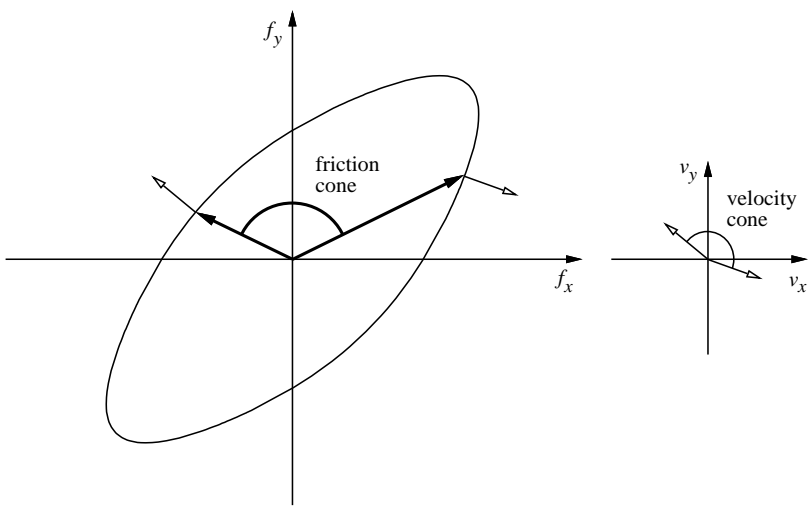

Figure 6: Mapping a friction cone $(\mu=2)$ to a velocity cone through the limit curve for $l=2$.

out the velocity cone. Note that the convexity of the limit curve implies that $\theta_{s}$ changes monotonically with $\phi$.

Now it is rather easy to see how pulling is possible. Coulomb's law is satisfied only by forces applied into the slider, but there is no similar requirement on velocities. For a large coefficient of friction, the velocity cone can include velocities away from the slider. Figure 6, for example, shows the velocity cone for $l=2$ and $\mu=2$. Note, however, that the quantity $\mathbf{f} \cdot \mathbf{v}_{s}$ must be nonnegative; that is, the pusher must perform nonnegative work in order to move the slider. Therefore, the velocity cone will always lie within 90 degrees of the friction cone.

\subsection{Slipping with infinite friction}

An infinite coefficient of friction results in a friction cone spanning 180 degrees, including exactly those forces directed into the slider. Due to symmetry in the limit curve, the velocity cone also spans 180 degrees. But unless this velocity cone is aligned with the friction cone, slipping may occur at the pusher-slider contact. Slipping occurs for any pusher velocity such that (1) $\mathbf{v}_{p}$ has a positive component in the contact normal $\left(v_{p y}>0\right)$, and (2) $\mathbf{v}_{p}$ is outside the velocity cone. The first constraint ensures that the slider must move in response to the push: the pushing constraint is active. The second constraint ensures that the slider velocity $\mathbf{v}_{s}$ is not equal to the pusher velocity $\mathbf{v}_{p}$ : the contact is slipping. For sticking contact to occur the fence would have to apply a force $\mathbf{f}$ away from the slider, which is impossible without adhesion.

Figure 7 shows the velocity cones for infinite $\mu$ and several choices of $l$. The pusher-slider contact will slip for $\theta_{p}$ just less than 180 degrees. Figure 8 demonstrates slipping contact for $l=5$. The rod endpoint velocity $\mathbf{v}_{s}$ cannot equal the pusher velocity $\mathbf{v}_{p}$ because $\mathbf{v}_{p}$ lies to the left of the velocity cone. Therefore, the contact is slipping
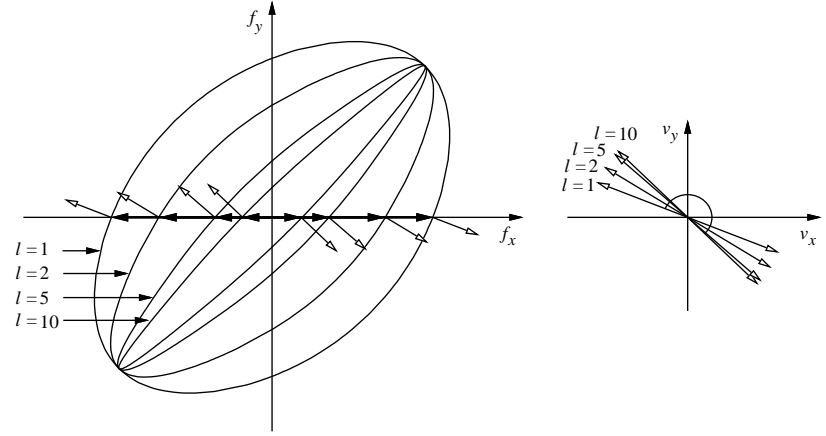

Figure 7: Infinite friction velocity cones.

to the right and the applied force $f$ lies on the left edge of the friction cone. This gives rise to a rod endpoint velocity $\mathbf{v}_{s}$ on the left edge of the velocity cone. The pusher, slider, and slipping velocities are illustrated in Figure 8.

Sticking contact will always occur for infinite $\mu$, regardless of the slider's support distribution, if the pusher moves exactly in the direction of the contact normal. The infinite friction velocity cone must lie within 90 degrees of the friction cone, and therefore the velocity cone must always include the contact normal. Slip is impossible for a normal push with infinite $\mu$ because it implies that the pusher performs negative work, i.e., $\mathbf{f} \cdot \mathbf{v}_{s}$ is negative.

\section{Dynamic pushing and pulling}

In this section we first analyze the dynamic motion of a slider on a frictionless support, and then we incorporate support frictional forces.

In the case of pure dynamic pushing, support frictional forces are negligible compared to inertial forces. It is a simple matter to show that the two effects demonstrated above for the quasi-static case also apply to the pure dynamic case. The example used here is analogous to that for the quasi-static case. The slider has unit mass uniformly distributed over the ring, giving it a unit radius of gyration. The linear acceleration of the pusher is $\mathbf{a}_{p}$ and the acceleration of the rod endpoint is $\mathbf{a}_{s}$. We will consider the initial motion of the slider starting from rest.

We will employ an acceleration cone, which is analogous to the velocity cone for quasi-static pushing. The forces which may be applied by the pusher, represented by the friction cone, map to a set of possible rod endpoint accelerations, represented by the acceleration cone. The acceleration cone possesses properties similar to those of the velocity cone. In particular, the angle of $\mathbf{a}_{s}$ changes monotonically with $\phi$, and $\mathbf{f} \cdot \mathbf{a}_{s}$ must always be positive, i.e., the acceleration cone always lies within 90 degrees of 


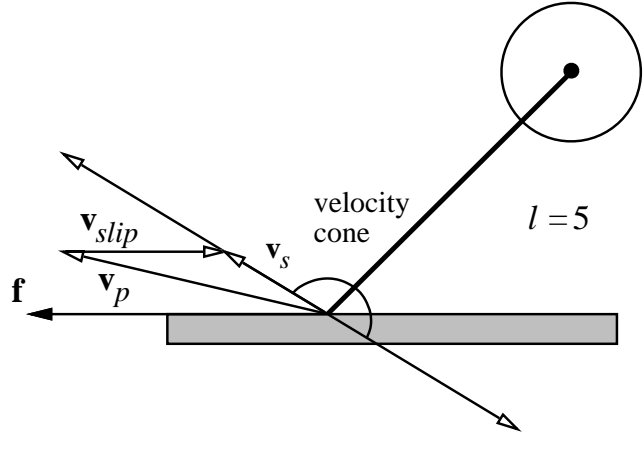

Figure 8: Slipping with infinite friction for $l=5$.

the friction cone. This is another way of saying that the mass matrix of the slider must be positive definite.

For a slider of unit mass, unit radius of gyration, and the general rod angle $\psi$, we find the generalized acceleration of the slider center of mass $\left(a_{c x}, a_{c y}, \alpha_{c}\right)$ :

$$
\begin{aligned}
a_{c x} & =f_{x} \\
a_{c y} & =f_{y} \\
\alpha_{c} & =\left(f_{x} l \sin \psi-f_{y} l \cos \psi\right)
\end{aligned}
$$

The rod endpoint acceleration $\mathbf{a}_{s}$ is then

$$
\begin{aligned}
a_{s x} & =a_{c x}+\alpha_{c} l \sin \psi \\
& =f_{x}\left(1+l^{2} \sin ^{2} \psi\right)-f_{y} l^{2} \sin \psi \cos \psi \\
a_{s y} & =a_{c y}-\alpha_{c} l \cos \psi \\
& =f_{y}\left(1+l^{2} \cos ^{2} \psi\right)-f_{x} l^{2} \sin \psi \cos \psi
\end{aligned}
$$

Pulling is possible when the rod endpoint acceleration is away from the slider $\left(a_{s y}<0\right)$, i.e., when

$$
f_{y}\left(1+l^{2} \cos ^{2} \psi\right)-f_{x} l^{2} \sin \psi \cos \psi<0
$$

Substituting $\mu f_{y}$ for $f_{x}$ and choosing $\psi=45^{\circ}$ and $l=2$, this condition becomes $3 f_{y}-2 \mu f_{y}<0$, which is satisfied for $\mu>1.5$.

Figure 9 shows the possible initial acceleration centers (found by the force-dual method of [3]) and the acceleration cones for $l=2$ and two different values of the coefficient of friction: (a) $\mu=2$; and (b) $\mu=\infty$. The pusher and slider are initially at rest. In Figure 9(a), pulling is a consistent solution if the pusher acceleration $\mathbf{a}_{p}$ is inside the acceleration cone and away from the slider $\left(a_{p y}<0\right)$. In Figure 9(b), slipping contact is the only solution if the pusher acceleration $\mathbf{a}_{p}$ is outside the acceleration cone with $a_{p y}>0$.

Continuing the analogy with the quasi-static case, we note that the mapping between the applied force angle $\phi$ and the angle of $\mathbf{a}_{s}$ is governed by an ellipsoidal surface analogous to the limit surface of the quasi-static case. An

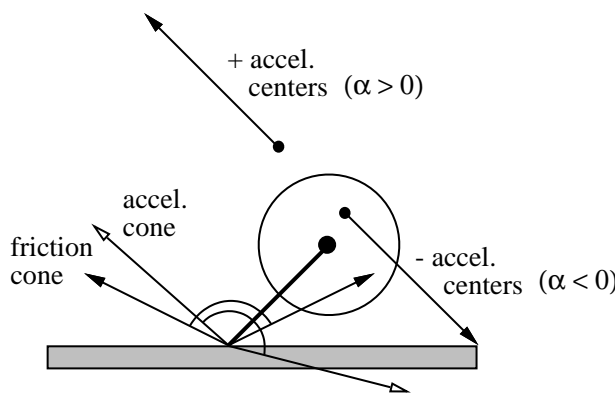

(a)

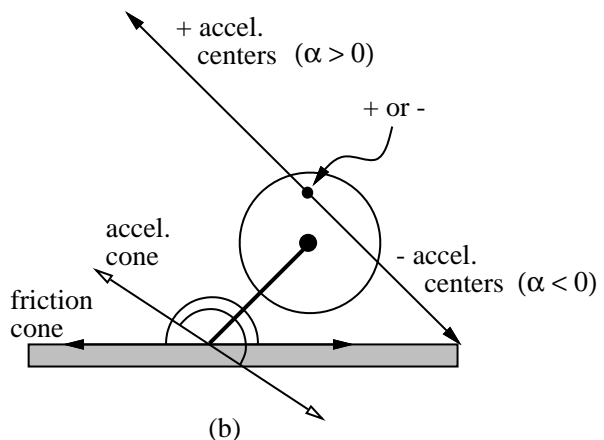

Figure 9: Acceleration cones for $l=2$. (a) $\mu=2$. (b) $\mu=\infty$.

applied force $\left(f_{x}, f_{y}, \tau\right)$ maps to an acceleration $\left(a_{x}, a_{y}, \alpha\right)$ normal to this ellipsoid at $\left(f_{x}, f_{y}, \tau\right)$. The $\tau_{0}=0$ plane of this ellipsoid is an ellipse with a major axis of half-length $c$ aligned with the rod and a perpendicular minor axis of half-length $c / \sqrt{1+(l / \rho)^{2}}$, where $\rho$ is the (unit) radius of gyration and $c$ is a scaling factor depending on the force magnitude. Figure 10 shows the ellipses corresponding to several rod lengths and the mapping of a friction cone to an acceleration cone for the case $l=1$. These ellipses clearly indicate the possibility of pulling and slipping with infinite friction.

We note that the dynamic pulling example is equivalent to a well known example of frictional ambiguity: a rod touching a nearly vertical wall in a gravity field. (See $[8,5,10]$.) As far as we know, no example of slip with infinite friction has previously been published.

Finally we address a case where neither friction nor dynamic forces can be neglected. In such a case neither the velocity cone nor the acceleration cone is directly applicable, but it is still easy to construct examples where the initial motion involves pulling or slip with infinite friction.

Again we consider the initial motion of an object starting from rest. The initial acceleration is represented by an acceleration center. This acceleration center is also the 


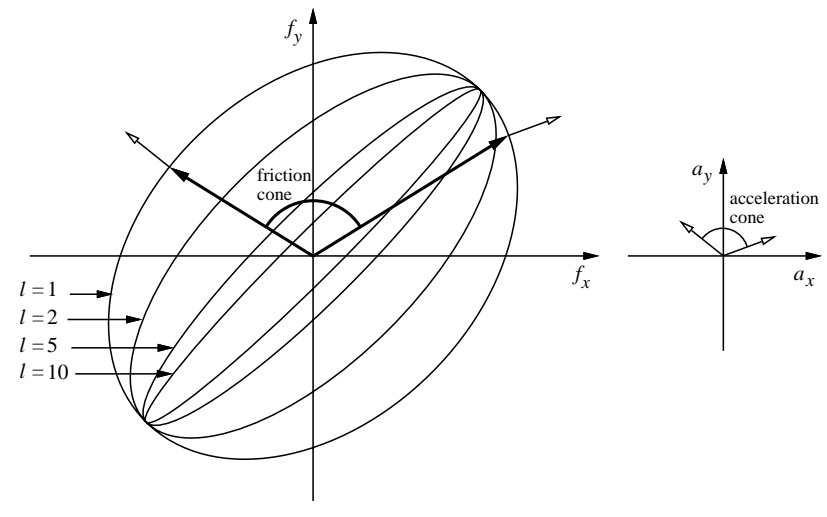

Figure 10: Ellipses governing the mapping from $\mathbf{f}$ to $\mathbf{a}_{s}$ for the pure dynamic case and four different rod lengths. Also shown is the mapping from a friction cone to an acceleration cone for $l=1$.

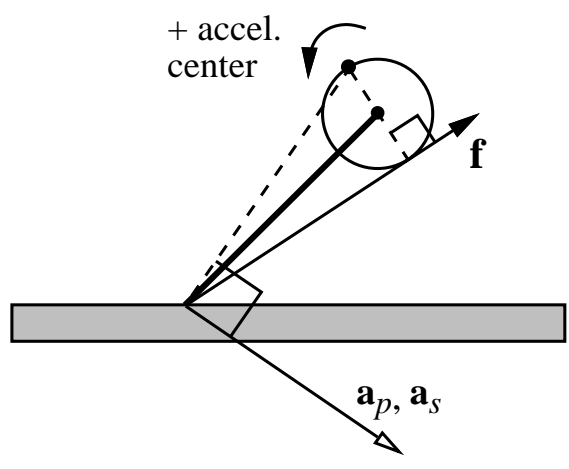

Figure 11: A pulling example, including support friction and inertial forces. $l=5$.

impending velocity center, from which we can derive the support frictional forces.

Figure 11 shows a possible pulling solution for $l=5$. Consider an initial acceleration center on the ring as shown. For this acceleration center, both the dynamic force and the frictional force act along a line through the contact point and tangent to the ring. Whatever their relative magnitudes, the resultant must act along the same line. A dynamic balance results from an applied force $f$ acting along the same line in the opposite direction, as shown. Notice that this acceleration center gives an initial rod endpoint acceleration away from the slider: pulling.

Demonstrating slip with infinite friction is a little more involved. We note the following geometrical relations that apply to the lines of action of both the support frictional force and the dynamic force:

1. Given an initial acceleration center with the slider initially at rest, each line of force is perpendicular to the line through the acceleration center and the slider center of mass.

2. Both lines of force are directed so as to oppose the acceleration.

3. Therefore, the two lines of force are parallel to each other, and to their resultant force.

4. Both lines of force lie on the opposite side of the ring center from the acceleration center.

5. If the acceleration center is outside the ring, each line of force passes through the ring.

6. If the acceleration center is on the ring, each line of force is tangent to the ring.

7. If the acceleration center is inside the ring, each line of force passes outside the ring.

Now we consider several cases, depending on the direction of the applied force. First, suppose the applied force acts within the friction cone, but along a line wholly to the right of the ring. Then both the support friction and the dynamic force must also pass the ring on the same side. Hence the acceleration center is inside the ring, on a line perpendicular to the applied force, and on the opposite side of the ring center. As we sweep the applied force through this range, the possible acceleration centers must fall within a sector of the ring interior.

Second, suppose the applied force is tangent to the ring. Both the support friction and the dynamic force must act along the same line. The acceleration center must lie on the opposite side of the ring.

Third, suppose the applied force passes to the right of the ring center, but through the ring. The support friction and the dynamic force must act on the same side of the ring center and through the ring. The acceleration center must lie on a line perpendicular to the applied force, on the opposite side of the ring center, and outside the ring. As the applied force is swept through this region, the acceleration center is confined to a sector of the plane outside the ring.

Fourth, suppose the applied force passes through the ring center. The support friction and the dynamic force cannot act on opposite sides of the center, so they must both act through the center. The acceleration center is at infinity, corresponding to a translation in the direction of the applied force.

There are three more cases, but they are similar to the first three. Taking all seven cases together, we find that the possible acceleration centers for $l=5$ are confined to the region shown in Figure 12. The corresponding initial accelerations of the rod endpoint are also shown. No initial acceleration outside that cone is possible. Slipping occurs 


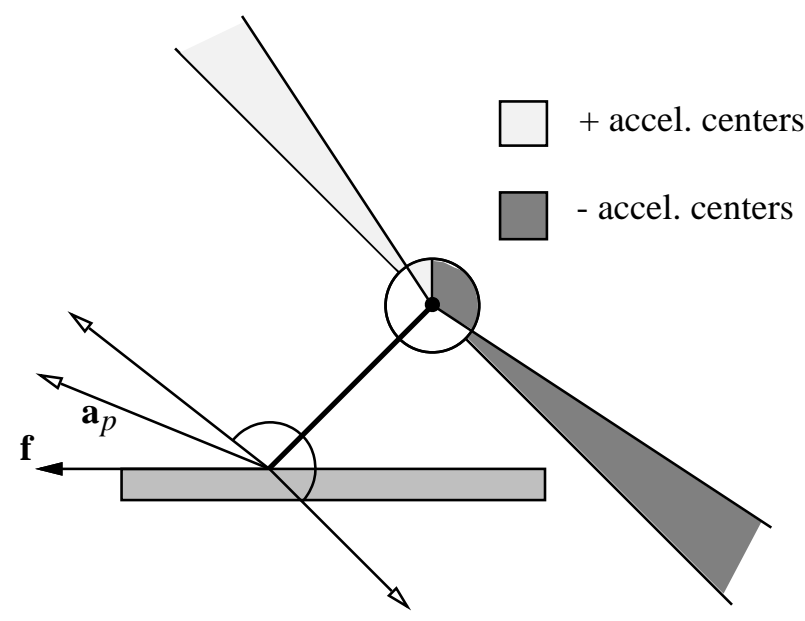

Figure 12: Slip with infinite friction, including support friction and inertial forces. $l=5$.

for an initial pusher acceleration $\mathbf{a}_{p}$ at angles just less than $180^{\circ}$, regardless of the magnitude of the acceleration. (Note that the bounds on the initial accelerations shown are a conservative approximation found using the geometrical facts given. Tighter bounds may be obtained with an exact analysis, but these bounds serve to demonstrate the phenomenon.)

\section{Remarks}

One of the difficulties in this work was that definitions of "infinite friction" and "perfectly rough" are generally imprecise and vary from one author to the next. In our sampling it appears that most authors really intend "perfectly rough" to mean a kinematic constraint that prevents slip, which is the definition we adopted. This definition does not need to be amended, but it is wise to bear in mind that it sometimes implies adhesive forces.

With respect to the notion of "infinite friction" the situation is less clear. It should be noted that slipping is possible with infinite friction because zero normal force is applied to the slider, thus allowing the tangential frictional force to be finite during slipping. Alexander and Maddocks [1] adopt a different definition of infinite friction which prevents a tangential force in the absence of a normal force. This definition changes the range of force angles which may be applied by the fence from the closed interval $\left[0^{\circ}, 180^{\circ}\right]$ to the open interval $\left(0^{\circ}, 180^{\circ}\right)$. These definitions of infinite friction correspond to (1) $f_{n}=0 \Rightarrow f_{t}$ unrestricted (and during slip, the frictional force is directed opposite the slipping velocity), and (2) $f_{n}=0 \Rightarrow f_{t}=0$, respectively, where $f_{n}$ is the normal force and $f_{t}$ is the tangential frictional force. In our examples, any situation in which definition 1 prevents sticking contact, definition 2 also disallows sticking contact. Definition 2, however, also disallows slipping contact; the slipping examples given above have no solution. Neither definition implements the perfectly rough surface of classical mechanics.

\section{Acknowledgments}

We thank John Maddocks for an interesting discussion on infinite friction and the members of the Manipulation Lab at CMU for their comments. This work was supported by NSF Grant IRI-9114208.

\section{References}

[1] J. C. Alexander and J. H. Maddocks. Bounds on the frictiondominated motion of a pushed object. International Journal of Robotics Research, to appear.

[2] D. Baraff. Coping with friction for non-penetrating rigid body simulation. Computer Graphics, 25(4): 31-40, 1991.

[3] R. C. Brost and M. T. Mason. Graphical analysis of planar rigid-body dynamics with multiple frictional contacts. In International Symposium on Robotics Research, pages 367374, Aug. 1989.

[4] P. E. Dupont. The effect of Coulomb friction on the existence and uniqueness of the forward dynamics problem. In IEEE International Conference on Robotics and Automation, pages 1442-1447, 1992.

[5] M. A. Erdmann. On motion planning with uncertainty. Master's thesis, Massachusetts Institute of Technology, Aug. 1984.

[6] S. Goyal. Planar Sliding of a Rigid Body With Dry Friction: Limit Surfaces and Dynamics of Motion. PhD thesis, Cornell University, Dept. of Mechanical Engineering, 1989.

[7] S. Goyal, A. Ruina, and J. Papadopoulos. Planar sliding with dry friction. Part 1. Limit surface and moment function. Wear, 143: 307-330, 1991.

[8] P. Lötstedt. Coulomb friction in two-dimensional rigid body systems. Zeitschrift für Angewandte Mathematik und Mechanik, 61: 605-615, 1981.

[9] M. T. Mason. Mechanics and planning of manipulator pushing operations. International Journal of Robotics Research, 5(3): 53-71, Fall 1986.

[10] V. T. Rajan, R. Burridge, and J. T. Schwartz. Dynamics of a rigid body in frictional contact with rigid walls. In IEEE International Conference on Robotics and Automation, pages 671-677, 1987.

[11] D. E. Rutherford. Classical Mechanics. Oliver and Boyd, London, 1951.

[12] Y.-T. Wang, V. Kumar, and J. Abel. Dynamics of rigid bodies undergoing multiple frictional contacts. In IEEE International Conference on Robotics and Automation, pages 2764-2769, 1992. 